\title{
Scaling and anisotropic heterogeneities of ocean SST images from satellite data
}

Francois G. Schmitt (1), Hussein Yahia (2), Joel Sudre (3), Véronique Garçon (3), Guillaume Charria (4), Boris Dewitte (3)

(1) CNRS-LOG, Wimereux, France; (2) GeoStat team, INRIA Bordeaux Sud-Ouest, France; (3) CNRSLEGOS, Toulouse, France; (4) Ifremer-LOPS, Brest, France

This belongs to the IMECO project, supported by the French LEFE-MANU programme
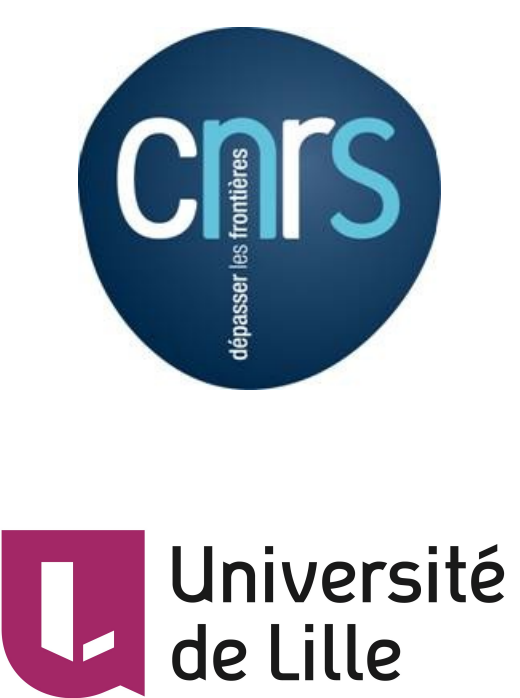

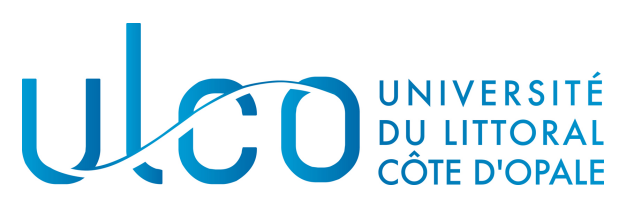

Oceanic fields display a large variability over large temporal and spatial scales. One way to characterize such variability, borrowed from the field of turbulence, is to consider scaling regimes and multi-scaling properties.

He we use 2D power spectral analysis as well as $2 D$ structure functions $\left\langle|X(M)-X(N)|^{q}>=F(q, d(M, N))\right.$, between two points $M$ and $N$ belonging to the region of interest. By performing statistics with respect to the distance $d(M, N)$, one may extract the scaling property of the $2 D$ field, for a range of distances $L_{\text {min }}<d<L_{\text {max }}$, of the form $F(q, d)=d \zeta(q)$. This approach can be used even for irregular images (having missing values due to cloud coverage) or for part of images in order to estimate the statistical heterogeneity of different zones of a given image.

In the framework of the French CNRS/IMECO project, we consider MODIS Aqua SST images, in France (English Channel versus Bay of Biscay) and in Chile (Eastern Boundary Upwelling System). We illustrate the use of the 2D structure function analysis for different parts of these images and also different times. Scaling ranges and also scaling exponents are compared. To take into account the anisotropy of some of these zones, an anisotropic version of the $2 \mathrm{D}$ structure functions is also used.
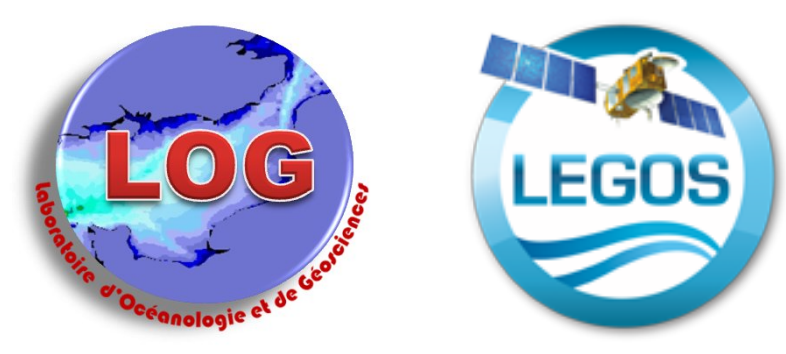
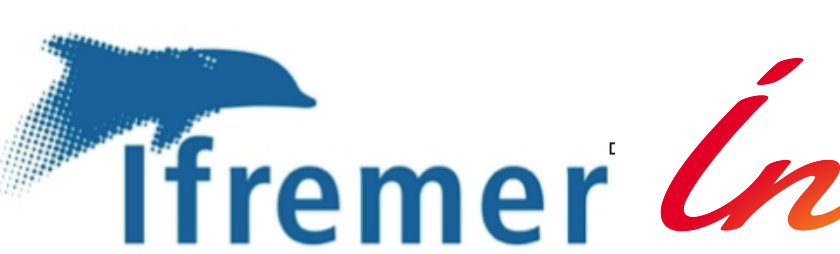


\section{Objectives}

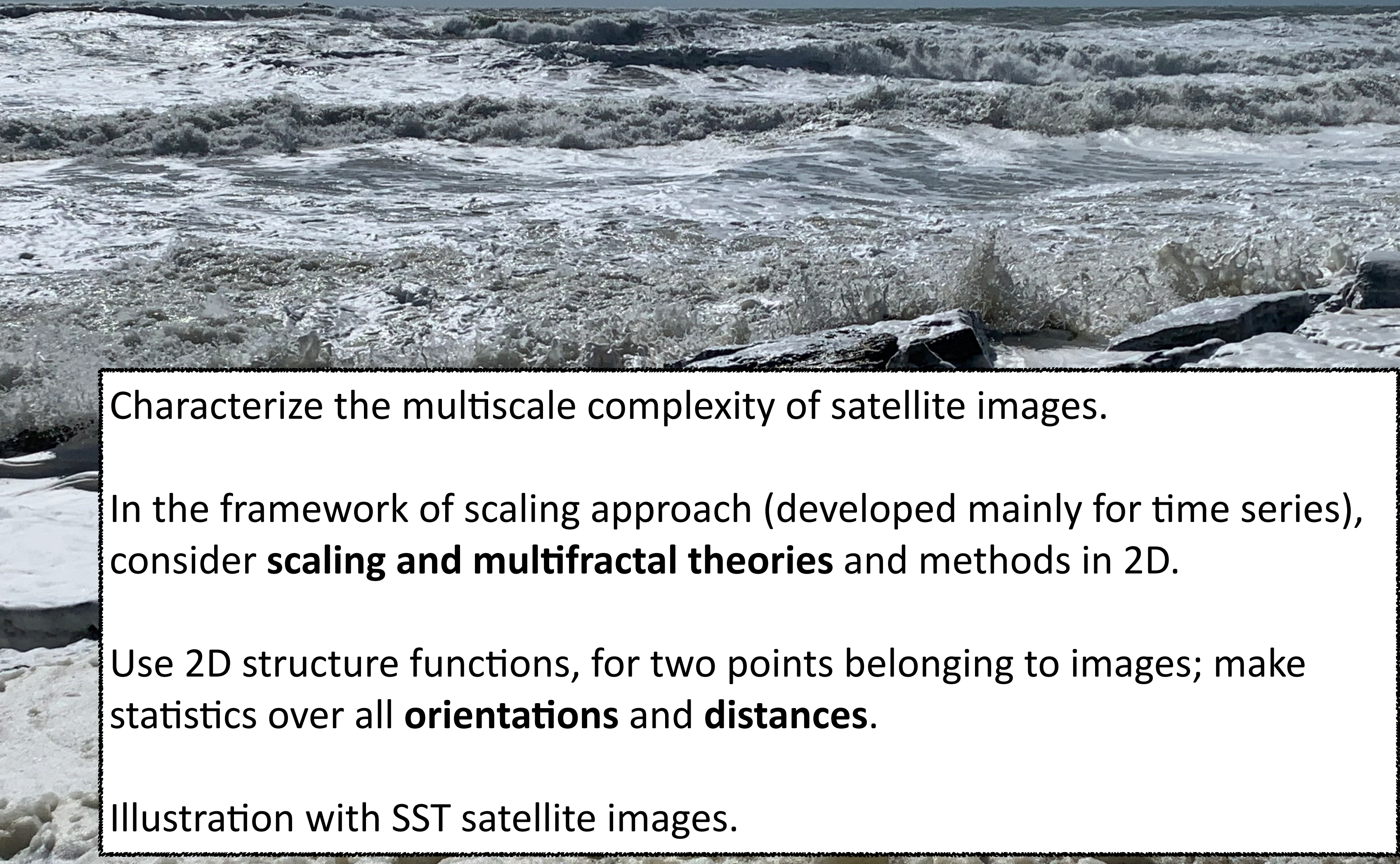




\section{Method}

In turbulence, use of structure functions since the 1940s (Kolmogorov 1941) Applied to time series

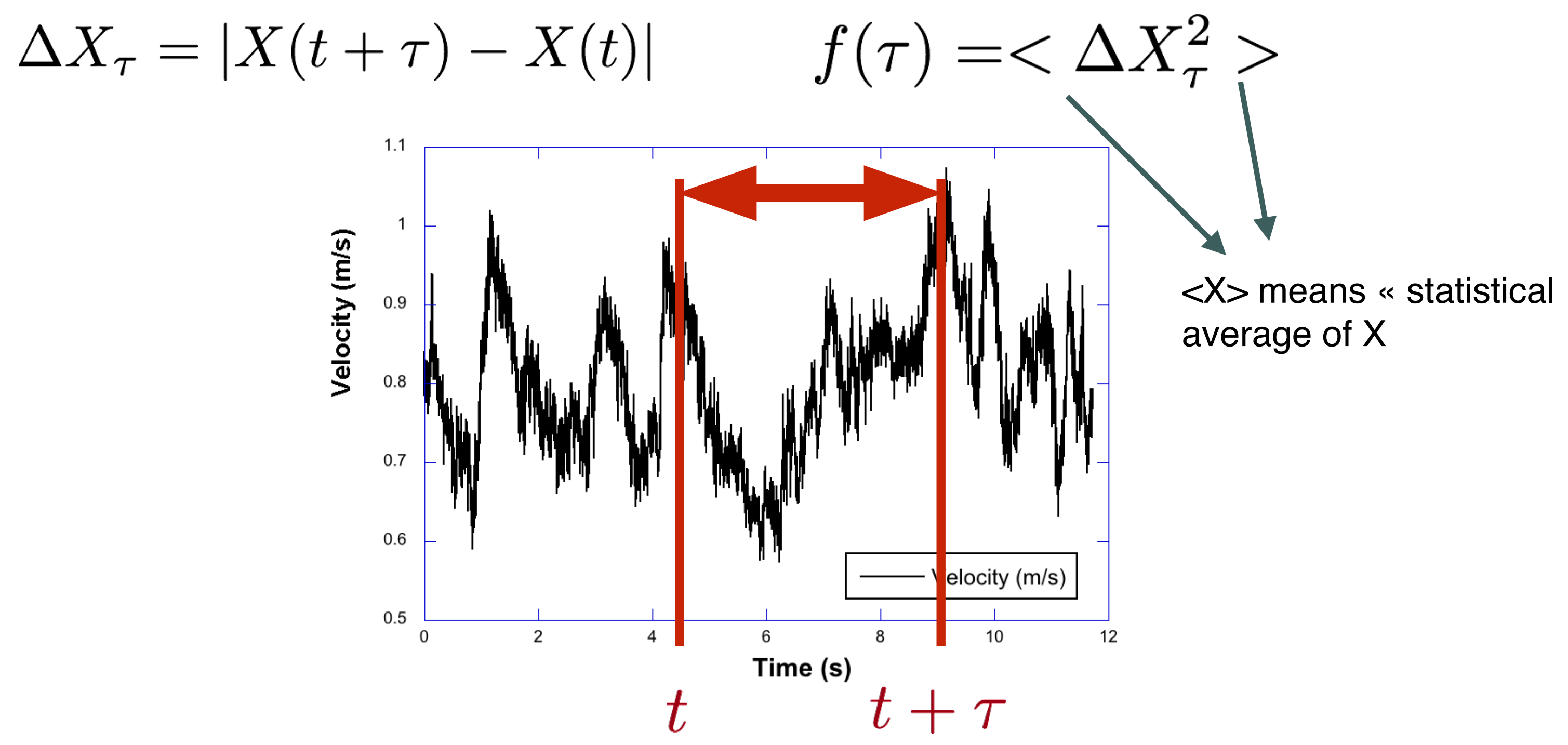

Characterizes the scale dependence of fluctuating fields. Applies to non-stationary series with stationary increments (often the case in geosciences) 


\section{Method}

$$
\Delta X_{\tau}=|X(t+\tau)-X(t)| \quad f(\tau)=<\Delta X_{\tau}^{2}>
$$

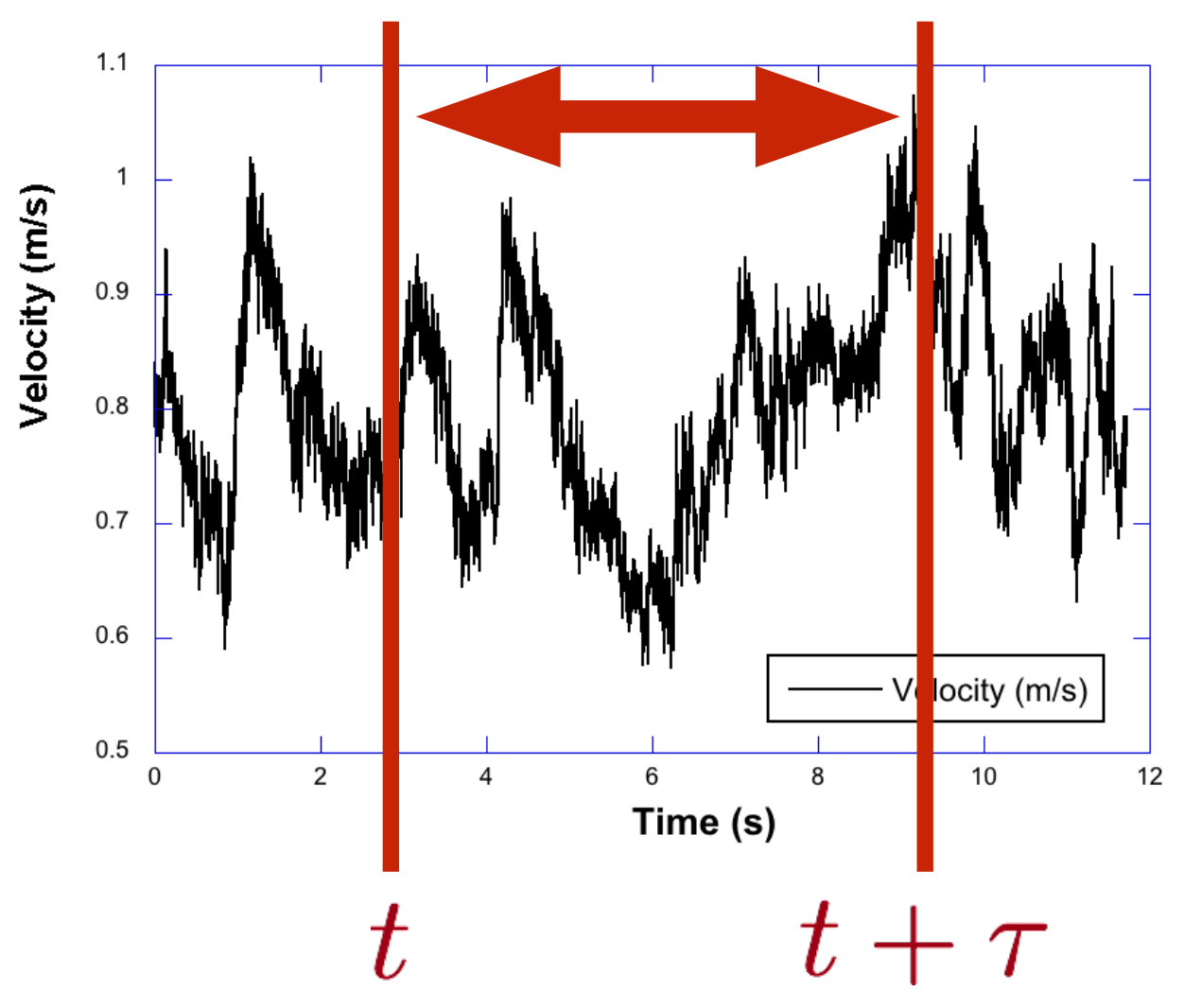

The following scaling property is very classical in turbulence (and many geophysical fields, temperature, pressure, humidity, ...)

$$
f(\tau)=<\Delta X_{\tau}^{2}>=C \times \tau^{b}
$$

$\mathrm{b}$ is estimated by a log-log plot of $f(\tau)$ versus $\tau$

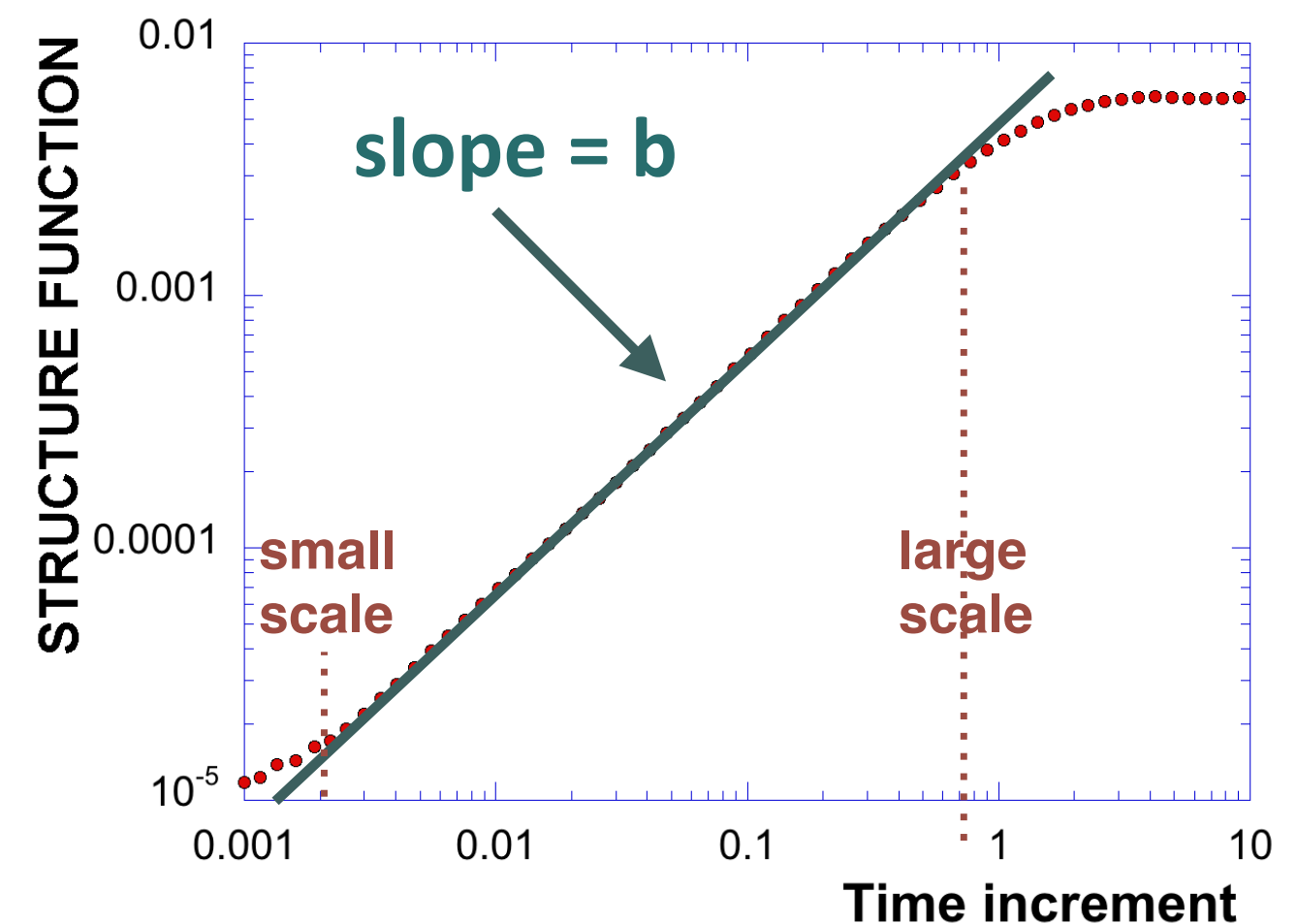




\section{Method}

$$
\begin{gathered}
\Delta X_{\tau}=|X(t+\tau)-X(t)| \\
f_{q}(\tau)=<\left(\Delta X_{\tau}\right)^{q}>q>0
\end{gathered}
$$

Generalization to moments of order $\boldsymbol{q}$

The larger $q$, the larger the fluctuations characterized

Example: $q=1$ medium fluctuations, $q=4$ large fluctuations 
Images: 2D structure functions also called " variogram " in the field of geosciences (only for moment $q=2$ ) (Matheron, 1963): geostatistics, soil studies, geomorphology.

Chase (1992), Lucazeau and Hurtez (1997), Western et al. (1998)

Distance dependence:

$f_{q}(d)=<|H(M)-H(N)|^{q}>$

In case of scaling property:

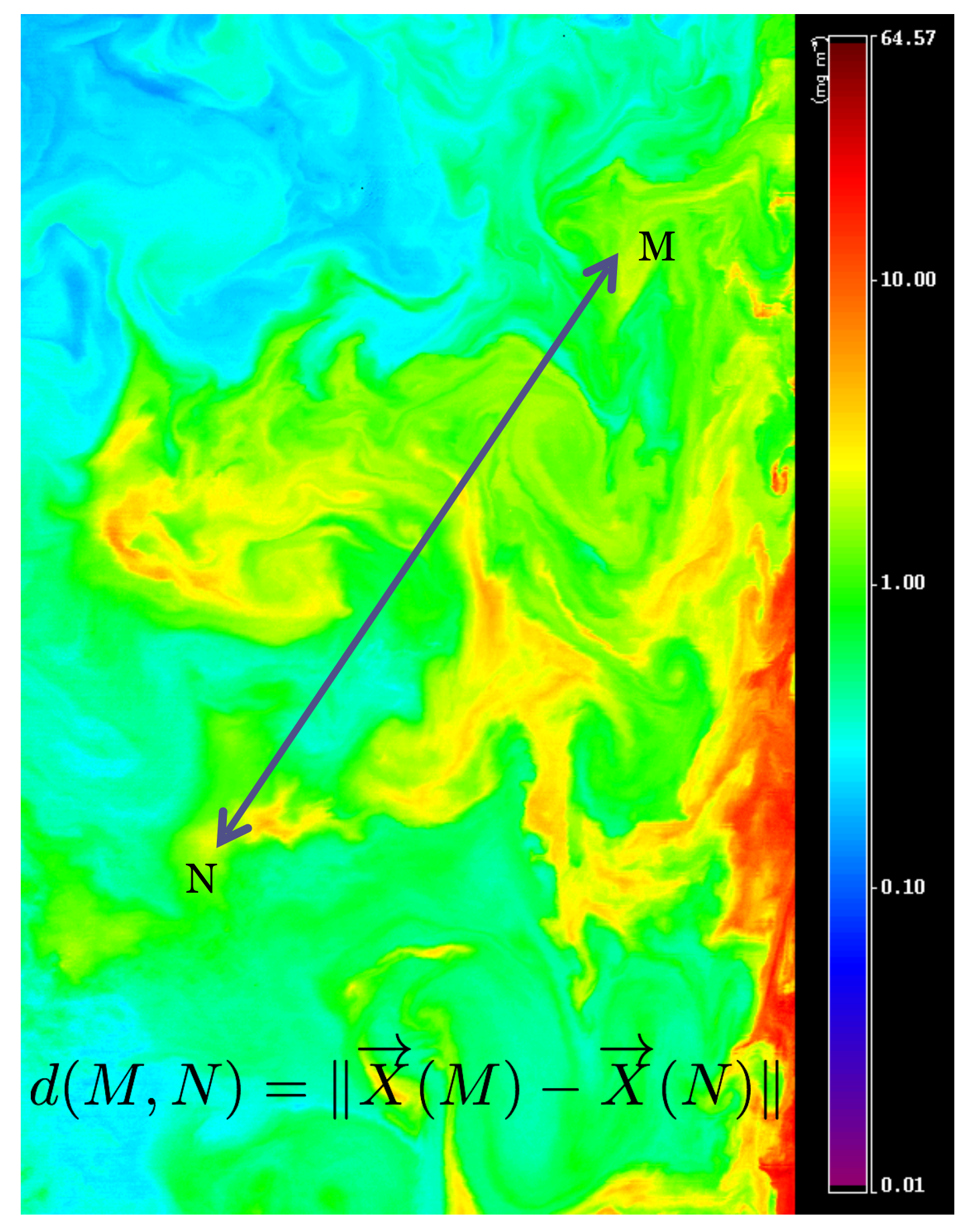

$$
f_{q}(d)=<|H(M)-H(N)|^{q}>=c_{q} \times d(M, N)^{\zeta(q)}
$$




\section{Advantages:}

- Can be used for irregular 2D fields

- Characterizes many scales and many intensities in the same time

- Global statistical characterization of the roughness of the data

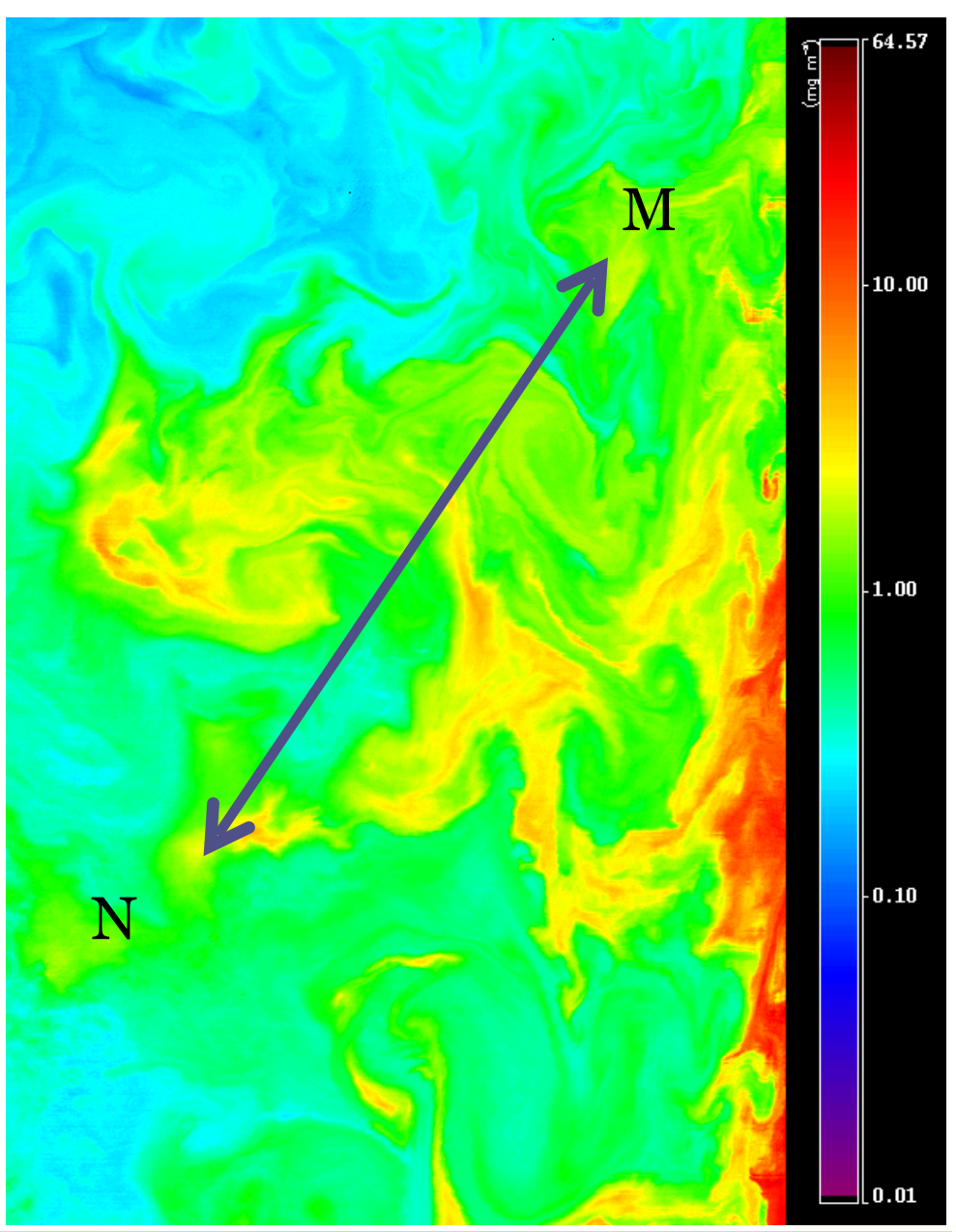

\section{Limits and methodology to overcome this limit:}

- for $1000 \times 1000$ images, $10^{6}$ points, $10^{12}$ couple of points: can be too large for computations;

- Choice of $\mathbf{1 0 0}$ millions of couple of points, chosen to have enough statistics for each distance: (i) random choice of a point $M$ in the domain; (ii) random direction; (iii) choice of the distance for the other point $\mathrm{N}$ (power law statistics for the distance);

- Tested for convergence of statistical moments 


\section{Images considered}

We consider here SST images as methodological examples.

These are from MODIS Aqua satellite, and represent (left) an Eastern Boundary Upwelling Systems (EBUS) near the coast of Chile $\left(-275^{\circ} \mathrm{E}->290^{\circ} \mathrm{E}\right.$ and $\left.40.125^{\circ} \mathrm{S} \rightarrow 24.875^{\circ} \mathrm{S}\right)$, and (right) the French Bay of Biscay of the Atlantic Ocean and the Channel $\left(350^{\circ} \mathrm{E}->3^{\circ} \mathrm{E}\right.$ and $41.875^{\circ} \mathrm{N}->$ $\left.52.125^{\circ} \mathrm{N}\right)$. Resolution: $4 \mathrm{~km}$. Below the scale is in pixels
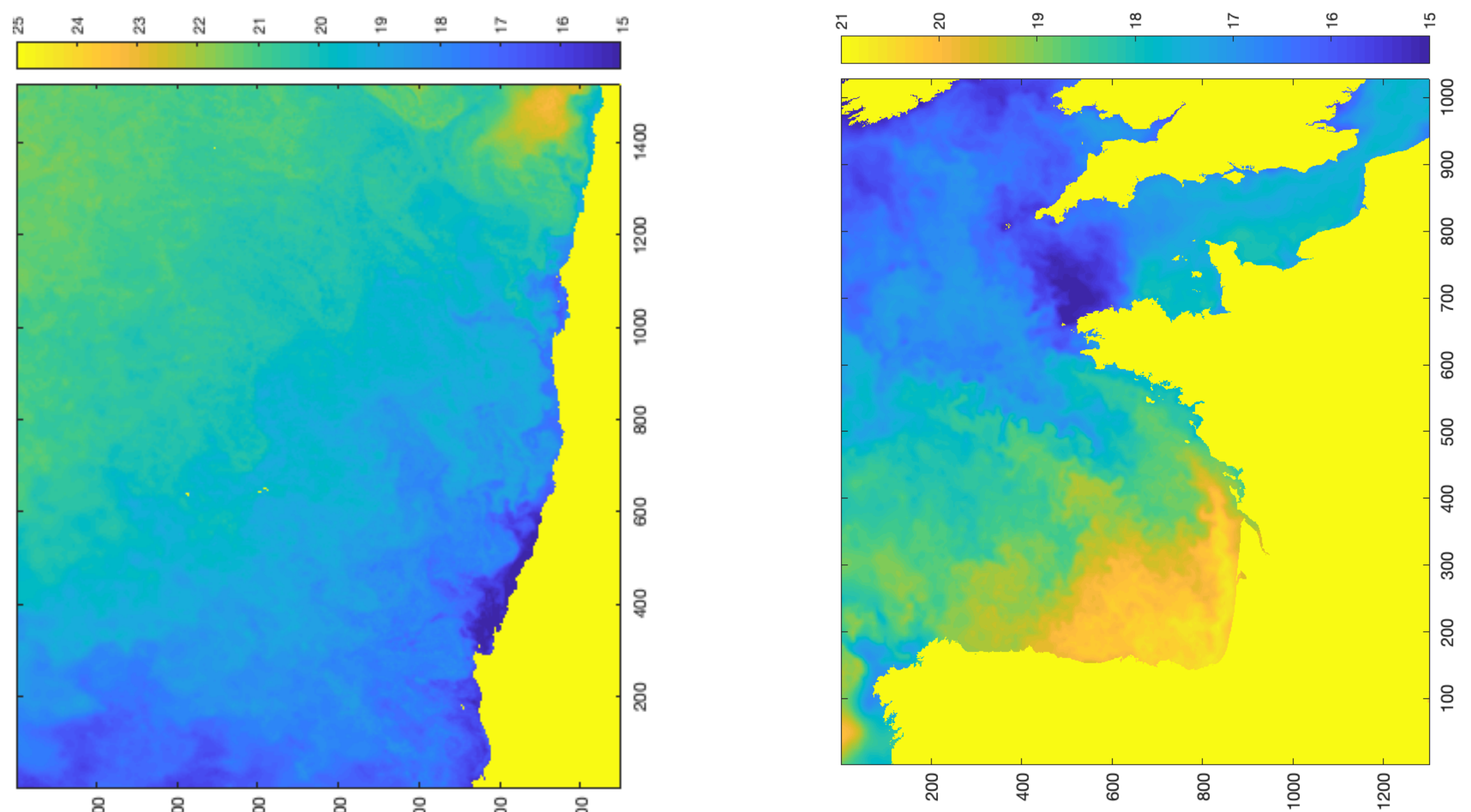


\section{Preliminary results}

For an image of size $1000 \times 1000$ pixels, there are $10^{\wedge} 12$ couple of points. This statistical approach is thus time consuming, but provides robust statistics.

Below one example performed on image SST2 (Bay of Biscay): statistical moments versus scale, where the scale is here the distance between points $M$ and $N$

$\stackrel{0}{0}$
$\stackrel{0}{0}$
$\stackrel{0}{0}$

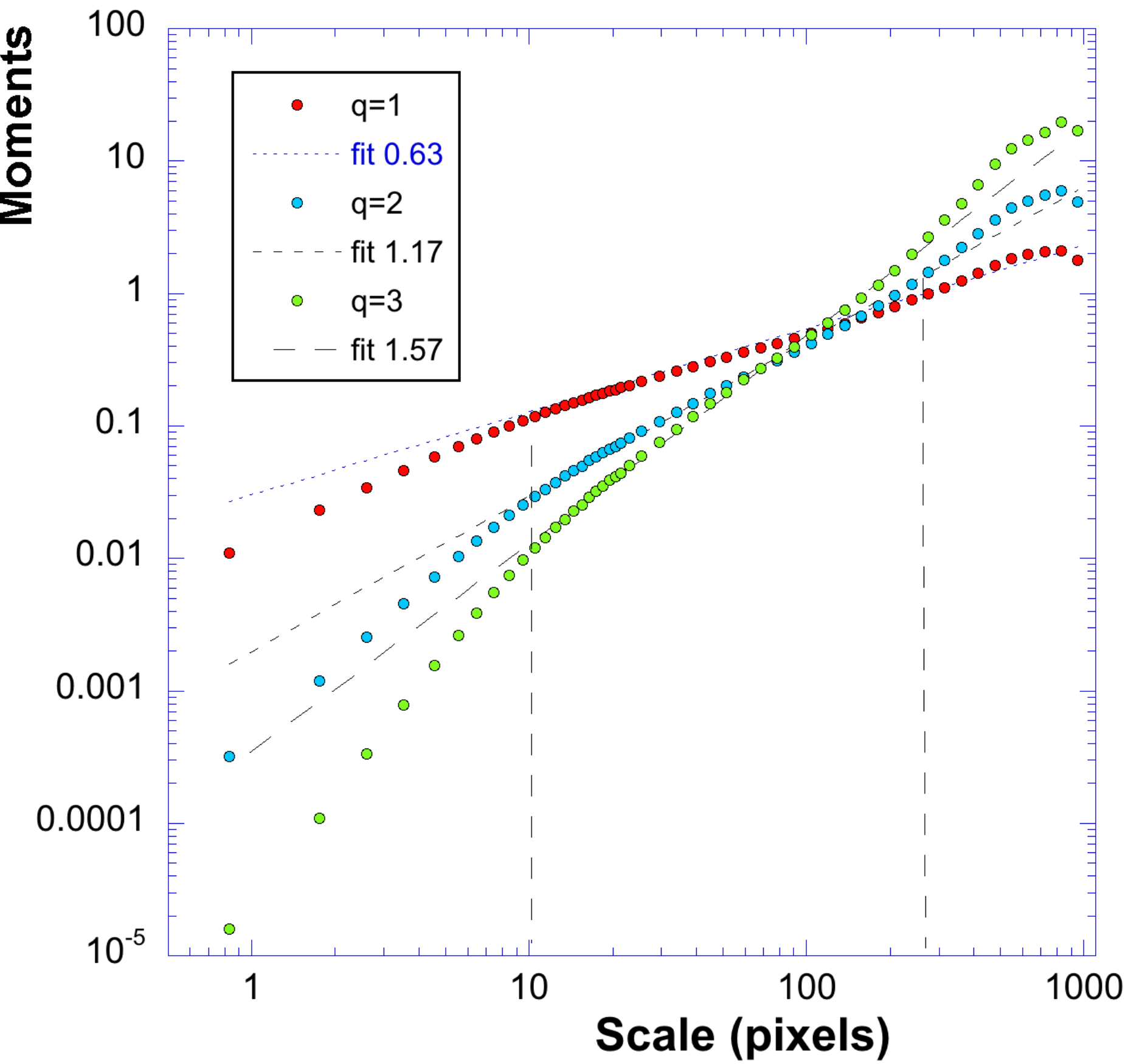

The scaling exponents are the slopes of the scaling curves estimated over the range between the two vertical dotted lines. 


\section{Preliminary results}

For an image of size $1000 \times 1000$ pixels, there are $10^{\wedge} 12$ couple of points. This statistical approach is thus time consuming, but provides robust statistics.

Below one example performed on image SST2: statistical moments versus scale, where the scale is here the distance between points $\mathrm{M}$ and $\mathrm{N}$

Multifractal scaling exponents for 2 SST images isotropic estimate

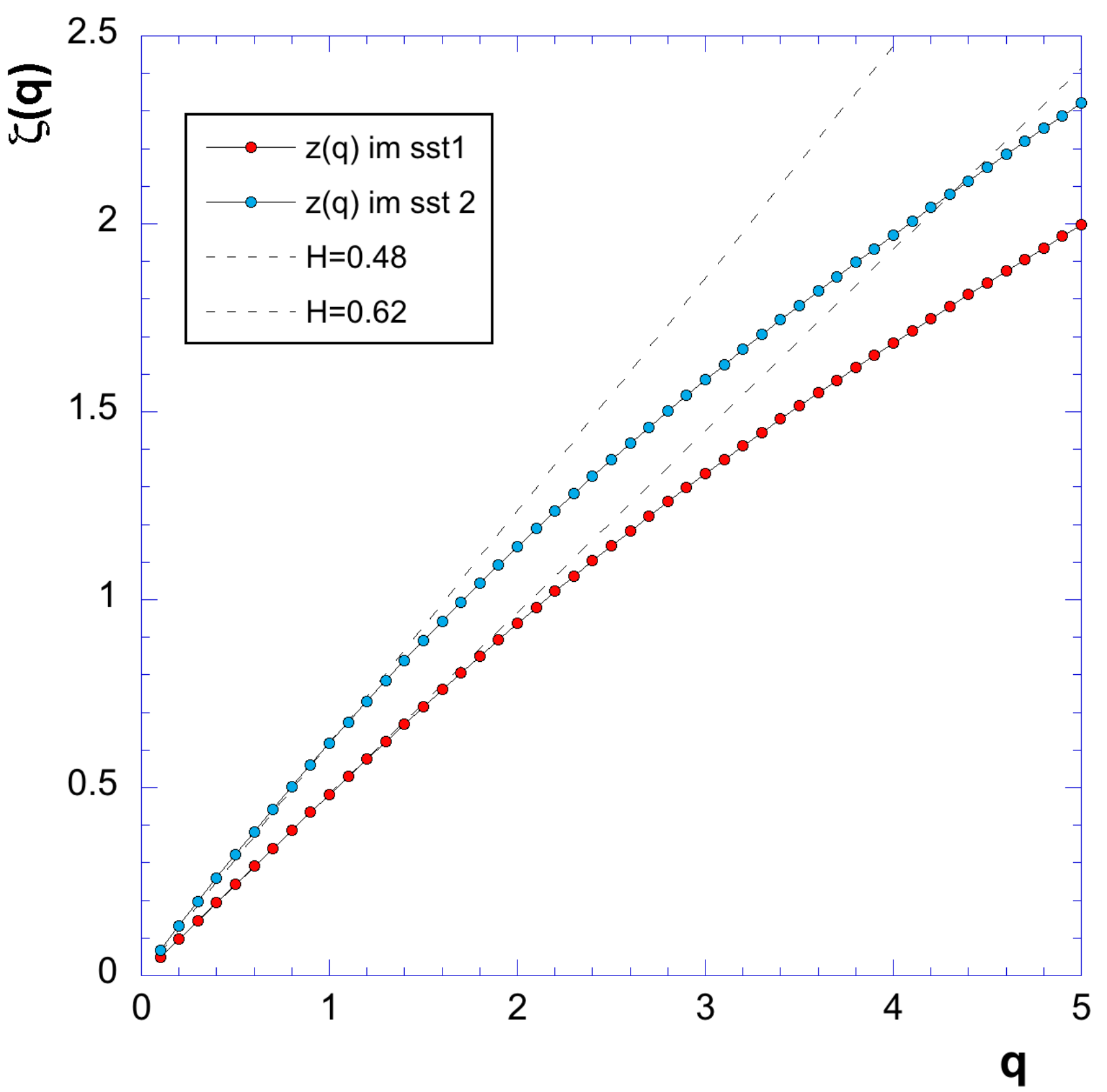

The scaling exponents estimated for images SST1 and SST2.

These curves are nonlinear and convex, as expected. The behaviours are similar, but the first moment $\mathrm{H}$ is larger for case SST2. This means that the corresponding field is smoother: SST field of Chile is smoother than French Bay of Biscay.

The convexity of the curves indicates that the fields are multifractal, i.e. a multiscale intermittent behaviour. 


\section{Preliminary conclusions}

Here we tested a simple generalization of the classical structure function framework which is classically used for the characterization of time series intermittency and multifractal properties.

The generalization is to do this in 2D, over a whole image, and all directions and all scales.

Two points $\mathrm{M}$ and $\mathrm{N}$ are chosen randomly in an image and the multiscale heterogeneity of the field studied is characterized using statistical moments of increments of the field, where the increment is the distance between the two points.

We obtained scaling properties, and scale invariant exponents are extracted over this range of scales. These exponents are used to compare intermittency properties of different images. For example we find similar intermittency (same concave shape of the exponents) but smoother behavior for Chile waters compared to Bay of Biscay. 


\section{Future work}

- Compare 2D structure functions and singularity exponents for a same image;

- Compare the multiscale properties of satellite images versus model outputs, for a same regional domain;

- Consider anisotropic structure functions exponents: the scaling is considered verbs the direction of the vector MN.

This helps to consider anisotropic scaling processes with different exponents associated with the direction of the vector composing the couple of points $M$ and $N$. The figure here shows an example using the two SST images, for the first moment. We see a smooth evolution of the angle with respect to the direction of the vector.

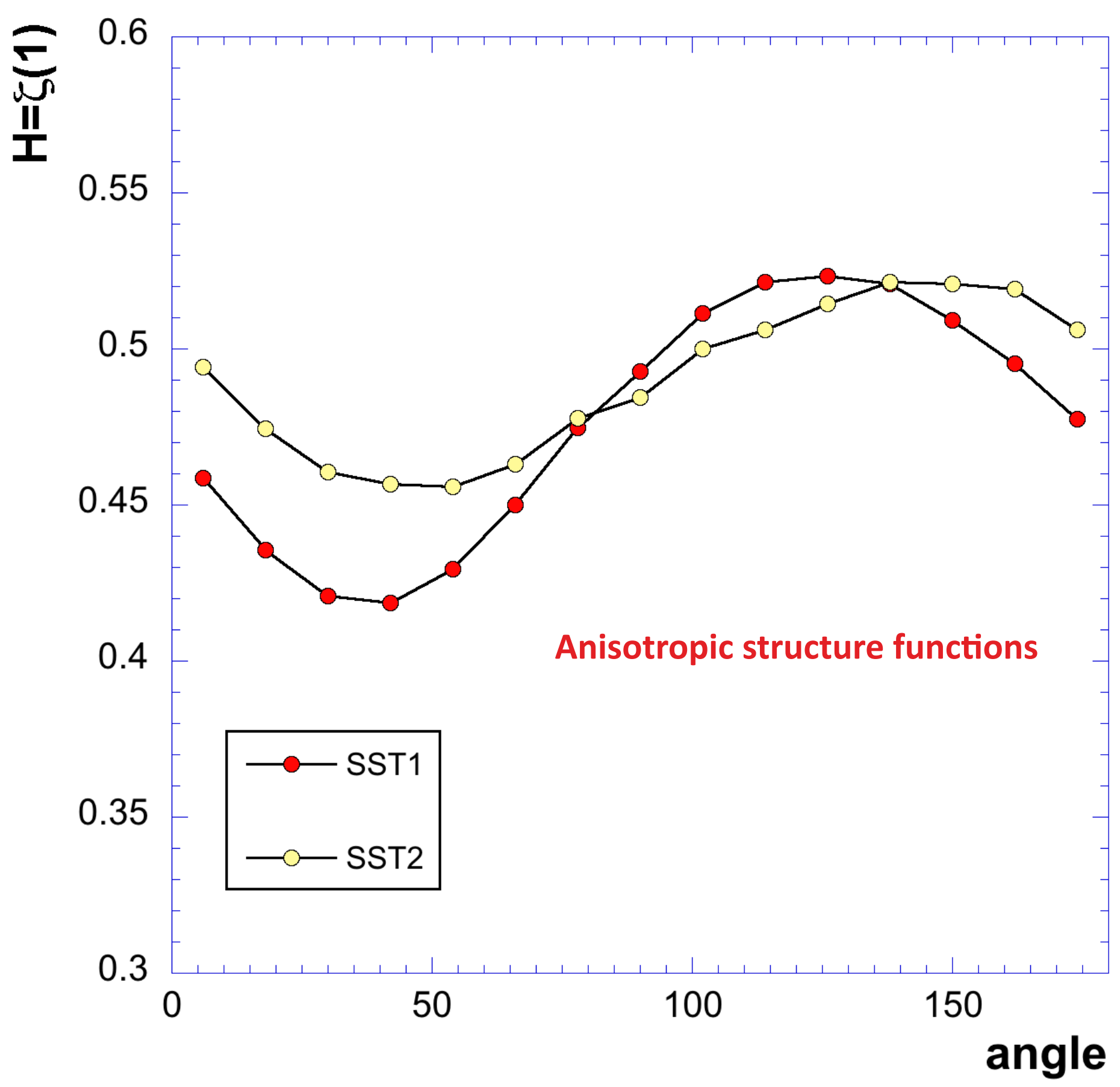




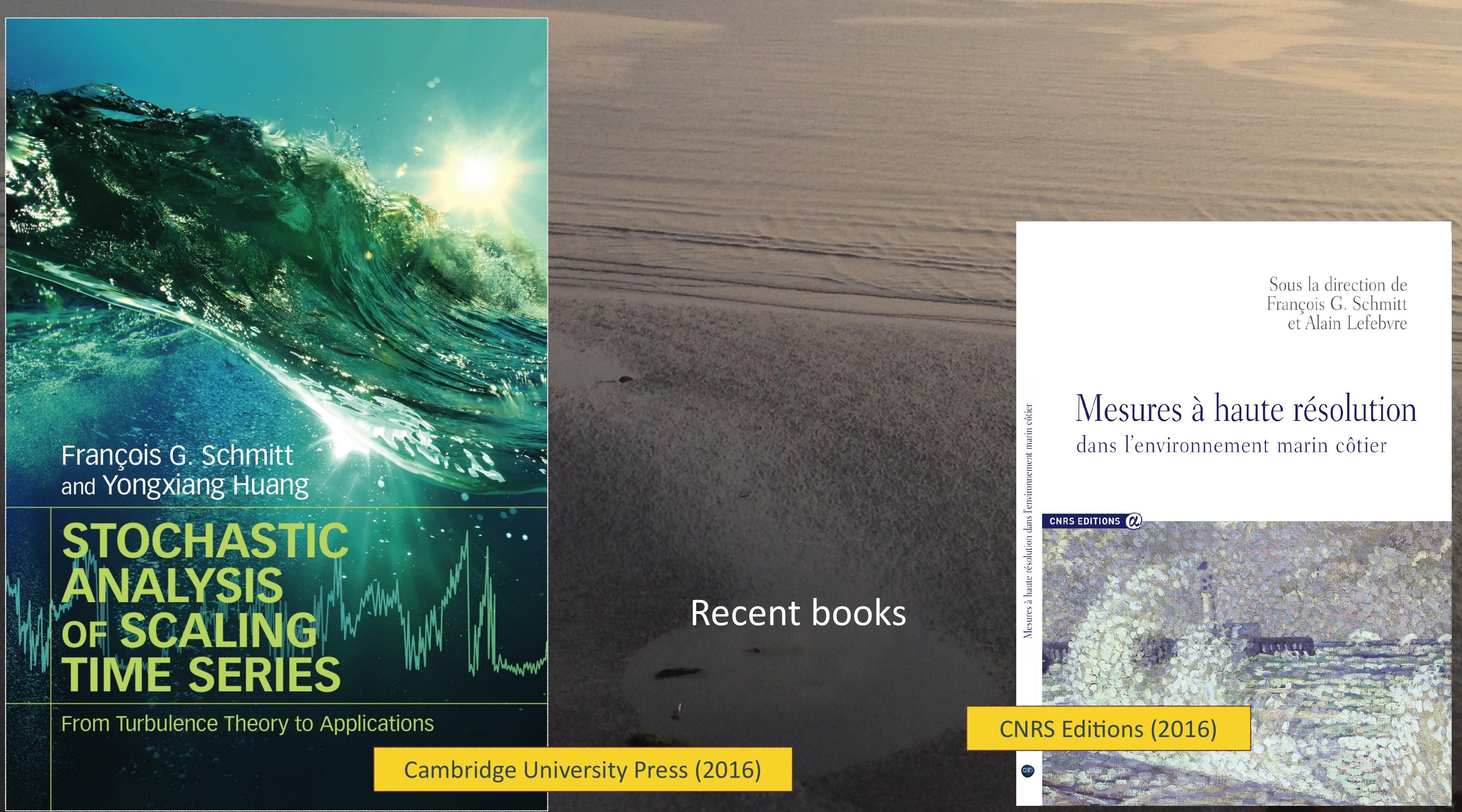

